\title{
Placental Hormone Activity Alteration
}

National Cancer Institute

\section{Source}

National Cancer Institute. Placental Hormone Activity Alteration. NCI Thesaurus. Code C40613.

Any physiologic process that results in modulated activity of any of the placental hormones, including choriogonadotropic hormone, chorionic somatomammotropin hormone, inhibin, estrogen and progesterone. These processes are involved in physiologic changes that support pregnancy. 